\title{
$\underline{\mathrm{M}^{\mathrm{a}} \text { Asunción Torres López }}$
}

Profesora Titular de Universidad Departamento de Derecho Administrativo

Universidad de Granada

\section{Unión Europea, Ciencia y Tecnología}

SUMARIO: I. LA POLÍTICA COMUNITARIA DE INVESTIGACIÓN Y DESARROLLO TECNOLÓGICO. 1. ETAPAS ESENCIALES DE LA EVOLUCIÓN HISTÓRICA DE LA POLÍTICA COMÚN DE IDT. 2. EL FUNDAMENTO JURÍDICO DE LA POLÍTICA COMÚN DE IDT. a) Objetivo de la política IDT y acciones propias de la Comunidad. b) Competencias de las Instituciones Comunitarias en materia de IDT. c) El instrumento principal de la política comunitaria de IDT. II. EL ESPACIO EUROPEO DE INVESTIGACIÓN. 1. LA DISTINCIÓN ENTRE EL ESPACIO EUROPEO DE INVESTIGACIÓN Y el VI Programa Marco. 2. El Espacio Europeo de Investigación: La ComuniCACIÓN DE LA COMISIÓN AL CONSEJO Y AL PARLAMENTO Europeo, AL COMITÉ ECONÓMICO y Social y al Comité de las Regiones «HaCia un Espacio Europeo de LA INVESTIGACIÓN». a) ¿Qué es necesario? b) Obstáculos a la creación de un Espacio Europeo de la Investigación. c) Exigencias del Espacio Europeo de la Investigación. d) Instrumentos para la realización del Espacio Europeo de la Investigación. e) La dimensión regional del EEI. III. El VI PROGRAMA MARCO DE LA UNIÓN EUROPEA. 1. ÁREAS DE ACTUACIÓN. 2. LAS ÁREAS PRIORITARIAS DEL VI PM. 3. LOS INSTRUMENTOS DEL VI PM. a) Los Proyectos Integrados. b) Las Redes de Excelencia. c) La participación en los programas nacionales realizados conjuntamente de acuerdo con el artículo 169 TUE. 4. Los Programas Específicos del Programa Marco 2002-2006. IV. DOS AÑOS DESPUÉS DEL CONSEJO EUROPEO DE LISBOA. NECESIDAD DE REFORZAR EL EEI. V. REPERCUSIONES DE LA POLÍTICA IDT PARA EL DERECHO ADMINISTRATIVO. 1. LA MODERNIZACIÓN DE LAS Administraciones Públicas. 2. La organización administrativa. 3. La EDUCACIÓN SUPERIOR: LA FUNCIÓN DE LA UNIVERSIDAD.

\section{LA POLÍTICA COMUNITARIA DE INVESTIGACIÓN Y DESARROLLO TECNOLÓGICO}

La Comunidad Europea surge con el objetivo esencial de promover y conseguir, mediante el establecimiento de un mercado común y de

Documentación Administrativa / nº. 265-266 (enero-agosto 2003) 
una Unión económica y monetaria y utilizando como instrumento y medio políticas o acciones comunes, «un desarrollo armonioso, equilibrado y sostenible de las actividades económicas en el conjunto de la Comunidad, un alto nivel de empleo y de protección social, la igualdad entre el hombre y la mujer, un crecimiento sostenible y no inflacionista, un alto grado de competitividad y de convergencia de los resultados económicos, un alto nivel de protección y de mejora de la calidad del medio ambiente, la elevación del nivel y la calidad de vida, la cohesión económica y social y la solidaridad entre los Estados miembros» ${ }^{1}$.

Todas las distintas políticas comunes se encuentran al servicio de tales fines, así como de las concretas libertades básicas. Todas ellas han ido creciendo y ampliando sus contenidos a lo largo de los años, incorporándose, tras las modificaciones de los Tratados, otras nuevas políticas en función de las necesidades no sólo de la industria sino también de las necesidades y exigencias de las sociedades europeas. Alcanzar estos objetivos exige la aplicación y perfección de instrumentos jurídicos y financieros concretos que permitan un desarrollo coherente en todos los países miembros.

Entre estas políticas tiene singular importancia e interés la llamada política de investigación y desarrollo tecnológico ${ }^{2}$ que, aunque contemplada en los Tratados originales, se ha ido perfeccionando hasta adquirir en la actualidad unas dimensiones asombrosas acorde con la imparable y espectacular revolución tecnológica a la que asistimos en los últimos años.

\section{ETAPAS ESENCIALES DE LA EVOLUCIÓN HISTÓRICA DE LA POLÍTICA COMÚN DE IDT}

Los antecedentes históricos de la política común de IDT se remontan a los años cincuenta, al origen mismo de los Tratados Comunitarios, con el establecimiento de la Comunidad Económica Europea (CEE) y de la Comunidad Europea de la Energía Atómica (CEEA); aunque, fruto del continuo progreso tecnológico, acentuado en los últimos años, ha ido perfeccionándose hasta adquirir una importancia vital entre las prioridades de la Unión Europea. La evolución de esta política ha sido ampliamente tratada en el estudio «¿Por qué cambian las políticas? La política europea de investigación y desarrollo tecnológico» $^{3}$, en el que se analizan las transformaciones en las políticas euro-

1 Artículo 2 Tratado de la Unión Europea.

2 Investigación y desarrollo tecnológico: IDT, en adelante.

3 Su autor es Luis SANZ MenÉndeZ, publicado en Revista Española de Ciencia Política, no. 4 (2001), pp. 97 a 121. Puede verse también A. CALONGE VelázQueZ, Administración e Investigación, Ed. MacGraw Hill, 1996, pp. 163 y ss. sobre la investigación y el desarrollo en Europa. 
peas de IDT, así como las razones y circunstancias que explican esta evolución.

A efectos de lo que nos interesa, la evolución de la política común de IDT puede concretarse en las siguientes etapas:

1) Hasta la década de los ochenta, la política común de IDT desde los años cincuenta no era más que la adición, si acaso, de las acciones desarrolladas en la materia por cada Estado miembro. La falta de un planteamiento europeo daba lugar tanto a repetición de trabajos como a pérdida de oportunidades.

La tendencia era abandonar la creación de Centros de Investigación comunes y acentuar la coordinación de las políticas nacionales y desarrollar la investigación cooperativa en el ámbito europeo entre las empresas y los centros de investigación existentes ${ }^{4}$.

2) Año 1984: se adopta el programa SPRIT (Programa estratégico europeo de investigación sobre tecnologías de la información), con el fin de fomentar la investigación básica sobre microelectrónica.

A partir de este año las actividades de investigación europeas y su financiación se apoyan en los denominados Programas Marco de investigación, desarrollo tecnológico y demostración, de carácter plurianual ${ }^{5}$.

3) Año 1986: con la aprobación del Acta Única Europea, la ciencia se sitúa entre las responsabilidades comunitarias. Por primera vez se definen en el Tratado cuáles son los objetivos de la política de IDT y que el instrumento de desarrollo de la misma sería el Programa Marco de IDT y los programas específicos; es decir, con el AUE existe ya una base legal específica ${ }^{6}$ para las acciones de IDT de la Comunidad y con ello se canalizan

4 Como señala SANZ MENÉndeZ en su estudio, los modelos iniciales de la política de IDT de las Comunidades se centran en las áreas específicas del carbón, el acero, la agricultura y la energía nuclear, siendo los actores principales los Gobiernos nacionales, caracterizándose, pues, esta política por una intervención directa del Estado. Ya en los años 60, la ejecución directa de la investigación se hace a través del Centro Común de Investigación y por una orientación hacia la energía nuclear (p. 103). En los años 70 se amplía la agenda europea hacia otras políticas no consideradas en los Tratados originales, y se centra en los «programas tecnológicos»; se pasa de la ejecución directa de la investigación a las actividades indirectas, fruto de la aplicación del principio de subsidiariedad (pp. 105-107).

5 El primer Programa Marco fue para el período 1984-1987; el segundo para el período 1987-1991; el tercero para el período 1991-1994; el cuarto para el período 1994-1998; el quinto para el período 1998-2002; y el sexto, recién aprobado, lo es para el período 2002-2006.

6 Artículo 130F AUE. 
las acciones de investigación dispersas en varias Direcciones Generales, a través de un Programa Marco global.

4) Años 90: comienza una nueva etapa a la que podemos llamar la Era Neotécnica; una verdadera revolución tecnológica origen de la competencia mundial y que, en gran medida, va a acentuar la importancia de la política europea de IDT.

Tras la aprobación del Tratado de la Unión Europea, se amplía el papel de IDT en la Unión Europea. La Comisión publica entonces su Libro Blanco sobre Crecimiento, Competitividad y Empleo (1995), en el que destaca la necesidad de la investigación, la tecnología y las telecomunicaciones, elementos impulsores para mantener la competitividad de la industria europea. En 1997 se aprueba el primer Plan de Acción sobre Innovación en Europa. Y con el Quinto Programa Marco (1998-2002) se pretende la concentración de recursos en menos programas, aunque, sin embargo, como indica SANZ MENÉNDEZ, los tradicionales proyectos de IDT han seguido dominando, ya que las ideas que formaban parte del nuevo paradigma de la innovación «no se abrieron paso en la acción política cuando entraron en conflicto con los intereses dominantes». En cierta manera tienen una explicación lógica, puesto que la instauración de ideas nuevas como es la innovación alteran los parámetros de esa política y provocan problemas por la resistencia de los países y de los intereses especiales a los cambios radicales en los objetivos, en los procedimientos y en la asignación de fondos ${ }^{8}$.

Precisamente, la asignación de fondos, al ser la política de IDT una política concebida como acciones distributivas, representa un problema interno a la hora de adoptar el Programa Marco.

Con el Tratado de Amsterdam ${ }^{9}$ las reglas de aprobación del Programa Marco cambian, al adoptarse el procedimiento de codecisión por mayoría cualificada del Consejo y el Parlamento Europeo ${ }^{10}$; y la Comisión continúa liderando las iniciativas.

5) Entrando en el siglo XXI, los esfuerzos comunitarios de IDT se centran fundamentalmente en la creación de un Espacio Europeo de la Investigación, eficaz y competitivo frente a los líderes

7 Si bien, con anterioridad, la Comisión aprueba una Comunicación en 1981 sobre la estrategia de desarrollo en IDT para los años 80 en la que propone al Consejo que todas las actividades de investigación y fomento de la IDT deberían incluirse en un Programa Marco global, algo que se conseguiría años más tarde con el AUE.

8 Véase SANZ MENÉNDEZ, op. cit., pp. 115-116.

9 En vigor a partir de 1 de mayo de 1999.

${ }^{10}$ Hasta entonces el Programa Marco era aprobado por el Consejo por unanimidad. 
mundiales, Estados Unidos y Japón, para aminorar los desequilibrios mundiales.

Como consecuencia del anterior postulado se crea una nueva política de IDT cuyos objetivos básicos son organizar la cooperación a distintos niveles; coordinar las políticas nacionales y europeas; fomentar la creación de redes de equipos de investigación y potenciar la movilidad de las personas y las ideas con el fin de fortalecer la competitividad de Europa.

\section{EL FUNDAMENTO JURÍDICO DE LA POLÍTICA COMÚN DE IDT}

«Fomento de la investigación y el desarrollo tecnológico». Con estas palabras se define en el artículo 3, letra n) del Tratado de la Unión Europea (TUE) la política común de IDT, que es desarrollada, tras las diversas modificaciones de los Tratados, en el Título XVIII bajo la rúbrica «Investigación y desarrollo tecnológico», artículos 163 a 173, y cuyo contenido pasamos a exponer brevemente, destacando las modificaciones habidas como consecuencia de la nueva política de IDT y su aplicación.

\section{a) Objetivo de la política IDT y acciones propias de la Comunidad}

La política comunitaria de IDT se erige fundamentalmente como una política de fomento, que con el fin expreso de «fortalecer las bases científicas y tecnológicas» de la industria comunitaria y favorecer el desarrollo de su competitividad internacional, trata de estimular a los tres agentes principales que en cada Estado miembro lideran la investigación en Europa, éstos son: las empresas, incluidas las pequeñas y medianas empresas ${ }^{11}$, en sus esfuerzos de investigación y desarrollo tecnológico de alta calidad; los Centros de Investigación y las Universidades.

Para ello se definen en el Tratado las acciones propias que la Comunidad puede llevar a cabo; en concreto son las siguientes:

1.- La ejecución de programas de IDT y de demostración, promoviendo la cooperación con empresas, centros de investigación y Universidades y de estas entidades entre sí.

11 Pequeñas y medianas empresas: PYMEs, en adelante. 
2.- La promoción de la cooperación en materia de IDT y demostración comunitaria con los terceros países y organizaciones internacionales.

3.- La difusión y explotación de los resultados de las actividades en materia de IDT y demostración comunitaria.

4.- El estímulo a la formación y a la movilidad de los investigadores de la Comunidad.

Estas acciones deben completar las acciones concretas que desarrollan los Estados miembros, de ahí la necesidad de coherencia recíproca de las políticas nacionales y de la política comunitaria, una exigencia de coordinación de acciones en materia de IDT que el propio Tratado impone $^{12}$, y sin la cual cualquier esfuerzo de unidad sería inútil.

\section{b) Competencias de las Instituciones Comunitarias en materia de IDT}

La iniciativa para promover la coordinación corresponde a la Comisión Europea y es el Consejo el que establece el Programa Marco plurianual que incluye el conjunto de acciones de la Comunidad.

El procedimiento es el establecido en el artículo 251 TUE: procedimiento de codecisión, que implica la adopción por mayoría cualificada del Consejo con el Parlamento Europeo y previa consulta al Comité Económico y Social.

La Comisión es también quien propone, para su aprobación por el Consejo y el Parlamento Europeo, los programas específicos desarrollados dentro de cada una de las acciones generales.

\section{c) El instrumento principal de la política comunitaria de IDT}

La política comunitaria de IDT se viene desarrollando a través de los denominados Programas Marco ${ }^{13}$, que se elaboran para períodos de cuatro años. Hasta ahora son seis los Programas Marco adoptados por la Comunidad. El último, el VI PM, se ha aprobado bajo la Presidencia española, para el período 2002-2006 y al que nos referimos más adelante. Con carácter general, los PM han de tener los siguientes contenidos:

1) Fijar los objetivos científicos y tecnológicos que deben alcanzarse y las prioridades.

\footnotetext{
12 Artículo 165 TUE.

13 Programa Marco: PM, en adelante.
} 
2) Indicar las líneas de dichas acciones.

3) Fijar el importe global máximo y la participación financiera de la Comunidad.

Los PM se ejecutan a través de programas específicos, desarrollados dentro de cada una de las acciones. Se prevé, asimismo, la elaboración de programas complementarios y de cooperación con terceros países, así como la posibilidad de crear empresas comunes u otro tipo de estructura necesaria para la ejecución de los programas de IDT y demostración comunitaria.

Finalmente, corresponde a la Comisión elaborar un Informe anual al Parlamento Europeo y al Consejo sobre las actividades realizadas en materia de IDT y la difusión de resultados. Las normas de participación son, en todo caso, fijadas por el Consejo.

\section{EL ESPACIO EUROPEO DE INVESTIGACIÓN}

\section{LA DISTINCIÓN ENTRE EL ESPACIO EUROPEO DE INVESTIGACIÓN Y EL VI PROGRAMA MARCO}

En enero de 2000, la Comisión presenta su propuesta «Hacia un Espacio Europeo de Investigación», donde señala las principales debilidades del sistema europeo con sus rivales principales: Estados Unidos y Japón.

El VI PM se inscribe en el EEI ${ }^{14}$. Son, pues, dos ámbitos distintos y dos iniciativas distintas, que es necesario diferenciar. No es de extrañar que una de las apreciaciones que el Estado español (Ministerio de Ciencia y Tecnología) hiciera a la Comunicación de la Comisión sobre la «realización del EEI: Orientaciones para acciones de investigación en la Unión Europea 2002-2006», es precisamente la falta de diferenciación clara entre el EEI y el VI PM.

El EEI es un proyecto con el que se pretende aumentar el impacto de los esfuerzos de investigación, reforzando la coherencia de las actividades y de las políticas científicas hoy vigentes en los países de la Unión Europea. El objetivo es construir un «mercado común de la investigación y la innovación», de la misma naturaleza que el mercado común de bienes y servicios.

EI VI PM que se inscribe en este proyecto es el instrumento normal de desarrollo de la política comunitaria de IDT y, como tal, define para

${ }^{14}$ Espacio Europeo de Investigación: EEI, en adelante. También conocido por las siglas ERA: European Research Area. 
el período 2002-2006 cuáles son las áreas prioritarias de investigación. En este sentido, la consideración del Programa Marco como el instrumento para asegurar la realización del EEI supera el carácter convencional de los Programas Marco anteriores, integrando las acciones comunitarias con las políticas nacionales y con los instrumentos disponibles en los programas nacionales.

2. El ESPACIO EUROPEO DE INVESTIGACIÓN: LA COMUNICACIÓN DE LA COMISIÓN AL CONSEJO Y AL PARLAMENTO EUROPEO, AL COMITÉ ECONÓMICO Y SOCIAL Y AL COMITÉ DE LAS REGIONES «HACIA UN ESPACIO EUROPEO DE LA INVESTIGACIÓN» ${ }^{15}$

En esta Comunicación la Comisión parte de la preocupante situación de Europa en cuanto al progreso de los conocimientos, y presenta como objetivo fundamental examinar cómo progresar en la dirección de una mejor organización de la investigación en Europa a través de la creación de un EEI. Un poco más tarde, la Comisión establece una serie de orientaciones para las acciones de la Unión en el ámbito de la investigación, completando así esta Comunicación ${ }^{16}$, que destaca cuatro puntos básicos de reflexión y propuestas:

a) En primer lugar, ¿qué es necesario? Destacamos brevemente las propuestas de la misma:

1.- Mejor inversión en conocimiento: su producción, su adquisición y su utilización.

2.- Mayor esfuerzo público de investigación, en tanto en cuanto la investigación tiene un papel esencial en la aplicación de las políticas públicas y ocupa un lugar importante en el proceso de decisión política.

3.- Promoción de las inversiones privadas en actividades de IDT.

4.- Mejor organización de la investigación en Europa, de manera que no se solapen las acciones nacionales y comunitarias y se integren o complementen, superando el actual esfuerzo investigador europeo que no es más que la adición de los esfuerzos de los Estados miembros y de la Unión, lo que origina la

15 COM (2000) 06, de 18 de enero de 2000.

16 Véase en la Comunicación al Consejo y Parlamento Europeo, al Consejo Económico y Social y al Comité de las Regiones COM (2000) 612 final, de 4 de octubre de 2000. 
fragmentación de los esfuerzos, el aislamiento, la compartimentación de los sistemas nacionales de investigación, y la disparidad de regímenes reglamentarios y administrativos.

5.- Creación de una verdadera política europea hacia un EEI que sea coherente en todo el territorio de la Unión Europea ${ }^{17}$.

b) En segundo lugar, ¿́cuáles son los obstáculos para la creación de un EEI?

1.- La compartimentación de los sistemas públicos de investigación.

2.- La falta de coordinación en la aplicación de las políticas nacionales y europeas.

3.- La existencia de barreras entre las distintas disciplinas, así como las que frenan la circulación de conocimientos y personas entre el mundo académico y el de empresas.

c) En tercer lugar, ¿̇en qué consiste un EEI, que permita las propuestas anteriores?

Teniendo en cuenta las consideraciones anteriores, el EEI exige:

1.- Un conjunto de recursos materiales e infraestructuras optimizado a escala europea: el establecimiento de una red de los centros de excelencia científica que existen en Europa y la creación de centros virtuales utilizando las nuevas herramientas de comunicación interactivas ${ }^{18}$; el enfoque europeo sobre las infraestructuras de investigación, cuyo problema fundamental es el del coste de construcción y de explotación, en ocasiones superior a las capacidades de un solo país; y la interconexión de las redes telemáticas nacionales ${ }^{19}$.

17 Precisamente éste fue el objeto de debate en el Consejo informal de Ministros de Investigación el 20 de mayo de 1999.

${ }^{18}$ Las redes electrónicas facilitan el trabajo a distancia, lo que permite la creación de verdaderos «centros virtuales de excelencia científica», multidisciplinares y que podrían asociar a Universidades y empresas.

19 En materia de redes electrónicas Europa va con retraso. De ahí la propuesta de la Comisión en la Cumbre de Helsinki de la iniciativa «E-Europa», en la que fija, entre sus objetivos de interconexión a escala europea, estimular la explotación máxima de estas redes por la comunidad de investigadores, tanto en el ámbito europeo como a nivel nacional: desarrollo de bases de datos y acceso a los servicios avanzados de Internet, estímulo a la producción de contenidos multimedia y utilizaciones interactivas, y apoyo a nuevos métodos de colaboración electrónica entre investigadores que prefiguren la aparición de verdaderos «institutos de investigación virtuales». 
2.- Unos instrumentos y medios públicos utilizados con coherencia: ejecución coordinada de los programas de investigación nacionales y europeos, con la aplicación del principio de apertura mutua de los programas nacionales. Este principio consiste en adoptar las medidas necesarias para garantizar su aplicación práctica, estableciendo mecanismos de información recíproca y un dispositivo de información general sobre los objetivos y el contenido de los programas y las condiciones de admisión y participación en los mismos.

3.- Una inversión privada más dinámica. En este sentido es necesario:

- Una mejor utilización de los instrumentos de apoyo indirecto a la investigación.

- Desarrollar herramientas eficaces para proteger la propiedad intelectual ${ }^{20}$.

- Estimular la creación de empresas.

4.- Un sistema común de referencia científico y técnico para ejecutar las políticas y que sea independiente de los intereses nacionales y privados. Propone la Comisión que el Centro Común de Investigación podría desempeñar un papel importante en el desarrollo de un espacio europeo de referencia científica y técnica, que se construiría sobre la base de los centros de referencia nacionales, los organismos europeos, los comités científicos y las instancias existentes en el ámbito europeo.

5.-Recursos humanos más abundantes y móviles ${ }^{21}$.

6.- Un territorio europeo más dinámico, abierto y atractivo para los investigadores del resto del mundo y las inversiones. Ello implica una especial atención a la participación de las Regiones en el esfuerzo europeo de la investigación y la innovación.

7.- Un espacio de valores compartidos. Esto es, que exista una visión común de las cuestiones de ética de la Ciencia y la Tecnología ${ }^{22}$; de las relaciones entre Ciencia y Sociedad en su dimensión europea.

${ }^{20}$ El actual Sistema Europeo de Patentes se basa en la expedición de patentes nacionales válidas sólo para los Estados miembros para los que se expiden; este sistema es costoso, lo que constituye uno de los principales obstáculos para su utilización en Europa. Por ello la propuesta de la Comisión es la adopción de una patente comunitaria única que cubra el territorio de la Unión.

21 Lo que implica: a) el aumento de la movilidad de los investigadores y la introducción de una dimensión europea en las carreras científicas; b) el refuerzo del estatuto y el papel de las mujeres en la investigación; c) el estímulo del gusto de los jóvenes por la investigación y las carreras científicas.

22 En ámbitos como las ciencias y tecnologías de la vida y las tecnologías de la información se plantean un gran número de cuestiones éticas. Y aunque los valores y principios fun- 
d) En cuarto lugar, ¿cuáles son los instrumentos para la realización del EEI?

El punto de partida es el principio de subsidiariedad en su sentido más amplio: las responsabilidades entre los Estados y la Comunidad se van a definir teniendo en cuenta el ámbito en que las acciones se pueden realizar con mayor eficacia.

Los instrumentos a disposición de la Unión Europea son:

1.- Instrumentos prácticos: bases de datos y sistemas de información.

2.- Mecanismos de intercambio de información y experiencias.

3.- Instrumentos financieros.

4.- Instrumentos jurídicos: Reglamentos y Directivas.

5.- Instrumentos de coordinación que faciliten el debate político y se concrete en una Recomendación o Resolución del Consejo.

e) La dimensión regional del EEI: Comunicación de la Comisión sobre la dimensión regional del Espacio Europeo de la Investigación de 3 de octubre de $2001^{23}$

¿Por qué son importantes las Regiones? Porque ellas constituyen el núcleo básico social, económico y cultural de los países con sus originales diferencias, y sin el establecimiento de medidas de fomento en los ámbitos regional y local, con la consiguiente configuración de los aspectos administrativos y organizativos que ello requiere, no es posible la realización del EEI.

A tal efecto destaca la Comisión en esta Comunicación las funciones que corresponden a las Regiones en el contexto general del espacio económico basado en la investigación, la tecnología y la innovación. Además, muchas Regiones europeas elaboran su propia política de IDT, de ahí el interés en promover la cooperación interregional a través de programas. Por ello la Comisión presenta diversas formas que permiten acentuar la importancia del papel de las Regiones en esta materia. Así, a través del fomento de las asociaciones entre el sector público y privado para contribuir a la economía europea del conocimiento; del

damentales son comunes en Europa, la manera de aplicar los principios es diferente, lo que supone que las cuestiones éticas vinculadas al progreso de los conocimientos científicos y tecnológicos den lugar a valoraciones diferentes según los países. Y si la discordancia es excesiva, no sería aceptable el respeto a las diferencias culturales y de sensibilidad moral que se derivan de esta variabilidad. De ahí la importancia de desarrollar enfoques convergentes y coherentes con estas cuestiones.

23 COM (2001) 549 final. 
estímulo de intercambio de experiencias con las Regiones que han tenido éxito en campos concretos; del establecimiento de estrategias de investigación e innovación para el desarrollo de recursos materiales y humanos como infraestructuras y equipos de investigación, instalaciones universitarias y de formación locales, estructuras de apoyo que fomenten la creación y el crecimiento de empresas innovadoras, etc.; de la contribución activa a la elaboración de una estrategia integrada para el desarrollo sostenible.

Pero todo ello exige un doble esfuerzo de coordinación, primero, en el ámbito interno de cada Estado miembro: política de IDT estatal-política de IDT regional; y, segundo, en el ámbito comunitario: política estatal de IDT-política común de IDT, que, a su vez, contemple la promoción de las Regiones. Una coordinación eficaz evitará duplicidad de acciones y un desarrollo armonioso del EEI desde el ámbito local-regional hasta el ámbito estatal en su integración en el ámbito europeo. Si bien existen pronunciamientos distintos que, al margen de la coordinación estatal-regional, buscan o pretenden que las Regiones europeas con competencias en materia de investigación dentro del Estado tengan una mayor representatividad en las instancias europeas. Es el caso de la Fundació Catalana per a la Recerca quien, en sus Recomendaciones en relación a la Comunicación de la Comisión sobre el EEI, afirma con rotundidad que debe dotarse de protagonismo y responsabilidad a las Regiones europeas, ya que son éstas las que se encuentran verdaderamente en contacto directo con la realidad de la actividad investigadora, y ello es posible eliminando las barreras que genera la propia organización del Estado ${ }^{24}$. Ello es cierto, pues la proximidad geográfica facilita los intercambios intelectuales, comerciales o financieros; las Regiones son la base territorial de las agrupaciones de investigadores, que pueden integrar distintos sectores: empresas innovadoras, universidades, centros de investigación, organismos de desarrollo locales y otras entidades de apoyo; integrando, igualmente, el sector público y privado ${ }^{25}$, en la medida en que ambos desempeñan funciones constructivas en este ámbito.

La coordinación se erige en problema por dos razones: primera, debido a las diferencias que aún existen en los ámbitos nacional y regional tanto en los aspectos tecnológicos y de innovación, como en recursos

${ }^{24}$ Véase en su página web: http://www.fcr.es.

25 Como expresamente señala la Comisión en la Comunicación citada, las agrupaciones más eficaces son las que cuentan con enlaces multisectoriales y organizaciones de distintos perfiles y en su forma más acertada combinan la industria, la administración pública y organizaciones no gubernamentales, además de otros agentes relacionados con la ciencia (universidades, centros de investigación, parques tecnológicos, polos de desarrollo tecnológico, entidades innovadoras que actúen como centros de servicios, y centros de competencia y difusión). 
humanos ${ }^{26}$. A tal efecto propone la Comisión intensificar la difusión de conocimientos y la modernización de los recursos humanos. Segunda razón, no todas las Regiones disponen de los mismos recursos humanos y materiales. De ahí que la participación de las Regiones menos favorecidas sea fomentada mediante la inversión en recursos a través del Programa Marco, de incentivos económicos, de creación de redes, etc.

Esta realidad que conduce a un planteamiento comunitario se incardina en el VI Programa Marco de IDT, que permite, a través de los nuevos instrumentos, el desarrollo de los recursos humanos en ciencia y tecnología y el desarrollo de la infraestructura científica en el contexto regional, así como el refuerzo de la dimensión regional y de la política comunitaria de desarrollo regional mediante una investigación apropiada.

En la valoración que el Comité de las Regiones da a la Comunicación de la Comisión «Hacia un Espacio Europeo de Investigación», en su Dictamen de 12 de abril de $2000^{27}$, afirma que los entes regionales y locales asumen funciones esenciales en la formación, el apoyo a los laboratorios y a las iniciativas de sus investigadores; y que, dentro del respeto al principio de subsidiariedad, la coordinación de los programas comunitarios dedicados a la investigación con las políticas regionales es fundamental para promover proyectos de desarrollo de la investigación que sean lo más próximos posible del ciudadano.

De este Dictamen existe otro aspecto que merece ser destacado, por insólito en los pronunciamientos de las Instituciones Comunitarias y nacionales sobre el Espacio Europeo de Investigación. Y es el recordatorio que hace en el punto 4 de que la investigación no se limita a las ciencias físicas y biológicas, sino también a las ciencias humanas y sociales. De ahí que una de sus propuestas sea la de respaldar desde el ámbito comunitario y de manera más significativa la investigación pura y la enseñanza superior dedicadas a las ciencias humanas ${ }^{28}$.

26 Las actividades se centran en los recursos inmateriales, más que en las infraestructuras físicas, y los Fondos Estructurales para el período 2000-2006 se dedican prioritariamente a la investigación, la innovación y la sociedad de la información y animan a las Regiones a adoptar vínculos de colaboración con las Universidades y las empresas.

27 COM-5/026, 24 de abril de 2000.

28 Los Estados miembros han manifestado su posición respecto de los programas específicos del Programa Marco, y debe destacarse, en la prioridad «Ciudadanos y gobernabilidad en la sociedad europea basada en el conocimiento», que, en general, requiere más elaboración; en particular, el Gobierno italiano indica la necesidad de añadir el «patrimonio cultural»; también Francia, quien desearía que se incluyese como programa específico el tema «la ciudad del mañana y su patrimonio cultural», pero, además, considera que la implicación de las ciencias humanas y sociales debe tratarse en estrecha conexión con los otros dominios prioritarios que afectan al ciudadano; el Gobierno noruego expresamente insiste en que la contribución de las Humanidades al desarrollo del EEI debería estar más claramente definida como parte de las prioridades de investigación, y que la importancia de perspectivas sociales y políticas debería también estar más claramente establecida tanto como un objetivo como en las prioridades de investigación concretas. 


\section{EL VI PROGRAMA MARCO DE IDT DE LA UNIÓN EUROPEA}

En febrero de 2000 la Comisión Europea presenta su propuesta de Programa Marco sobre la investigación y la innovación para el período 2002-2006. Un nuevo PM distinto a los anteriores, que incorpora nuevos instrumentos para su ejecución ${ }^{29}$.

\section{1. ÁREAS DE ACTUACIÓN}

Son tres las áreas de actuación principales en las que se integra el VI PM y que reflejan los intereses básicos del EEI:

1. «Integrar la investigación europea», lo que implica dos consecuencias esenciales: la primera, el establecimiento de un número limitado de áreas de investigación prioritarias; y la segunda, un refuerzo de los vínculos entre la política comunitaria de IDT y las políticas de investigación nacionales y regionales.

2. «Estructurar el EEI». Con el fortalecimiento de los vínculos entre investigación e innovación y con la renovación del potencial humano de investigación y la movilidad de los investigadores, el VI PM innova con un aspecto importante: el apoyo al desarrollo de las infraestructuras de investigación mediante acciones integradas que faciliten su puesta en red y la prestación de servicios científicos a nivel europeo.

3. «Reforzar las bases del EEI». El nuevo PM pretende fortalecer una de sus misiones fundamentales que es la de satisfacer las necesidades científicas y tecnológicas derivadas de la aplicación de las políticas de la Unión Europea en todos los ámbitos en que la Comunidad tiene responsabilidades crecientes ${ }^{30}$.

\section{2. ¿CUÁLES SON LAS ÁREAS PRIORITARIAS DEL VI PM?}

Son siete las áreas prioritarias:

29 Véase la Decisión No 1.513/2002/CE del Consejo y del Parlamento Europeo concerniente al Sexto Programa Marco de la Comunidad Europea para la investigación, el desarrollo tecnológico y las actividades de demostración, contribuyendo a la creación del Espacio Europeo de la Investigación y la Innovación (2002-2006), de 27 de junio de 2002, publicada en el DOCE de 29 de agosto.

El texto completo (versión en inglés) puede verse en la página web: http://www.sost.cdti.es/programaVI.htm.

30 Tales como agricultura, pesca, salud y protección del consumidor, medio ambiente, transporte y sociedad de la información. 
1. Genómica y Biotecnología ${ }^{31}$.

2. Tecnologías para la sociedad de la información ${ }^{32}$.

3. Nanotecnología y nanociencia ${ }^{33}$.

4. Aeronáutica y espacio ${ }^{34}$.

5. Seguridad alimentaria y riesgos de salud ${ }^{35}$.

6. Desarrollo sostenible y medio ambiente ${ }^{36}$.

7. Ciudadanos y gobernanza ${ }^{37}$.

A estas siete áreas se suma otra actividad llamada «Anticipar las necesidades científicas y tecnológicas de la Unión Europea», conocida como la octava prioridad $^{38}$. Éste es el mecanismo propuesto por la Comisión para incluir otras acciones no ligadas exclusivamente a las Áreas prioritarias. Si bien, debería abordarse desde un punto de vista amplio con el fin de incluir áreas de investigación básica sin ninguna restricción a priori y abierto a la iniciativa de los propios grupos de investigación ${ }^{39}$.

\section{3. ¿CUÁLES SON LOS INSTRUMENTOS DEL VI PM?}

Son tres los nuevos instrumentos propuestos por la Comisión:

a) Los proyectos integrados ${ }^{40}$, que incluyen la cooperación Universidad-Industria, con altos presupuestos

Éste es uno de los objetivos básicos de la política comunitaria, el acercar a través de la investigación dos ámbitos antagónicos, el acadé-

31 Proteómica, bioinformática, prevención y terapia; lucha contra las enfermedades, antibiótico, cáncer, sida.

32 Seguridad, comercio electrónico; comunicación, computación, programas.

33 Materiales inteligentes, arquitectura supramolecular, nanobiotecnología; materiales inteligentes y multifuncionales, nuevos dispositivos.

34 Nuevos aviones, motores, impacto ambiental, seguridad; satélites, vigilancia ambiental, telecomunicaciones.

35 Epidemiología y alergias, dieta, análisis y control; producción segura, salud humana y alimentación animal.

36 Energía limpia, ahorro, pilas de combustible, transporte limpio; emisiones, ciclo de agua, biodiversidad, modelos climáticos.

37 Sociedad del conocimiento y cohesión social; consecuencias de la ampliación, resolución de conflictos.

38 Documento Anticipating the EU's Scientific and Technological Needs. Operational Principles, que puede consultarse en la página web: http://sost.cdti.es/programa VI_1.htm.

39 Es ésta una de las apreciaciones o sugerencias que refleja el documento que recoge la posición común de las Universidades españolas frente a la propuesta de la Comisión Europea relativa al VI Programa Marco de I + DT, de 30 de marzo de 2001.

40 Puede verse el texto en inglés (Provisions for implementing Integrated Projets) en la página web: http://www.sost.cdti.es/programaVI_1.htm. 
mico y el empresarial, ambos ofrecen puntos de vista distintos pero a la vez complementarios, de ahí la importancia de facilitar el intercambio de los investigadores entre estos dos «cotos cerrados».

Los proyectos integrados son utilizados en las siete áreas prioritarias o también, si fuera relevante, en áreas de investigación que reúnen las necesidades de las políticas comunitarias, así como en nuevas y emergentes áreas del conocimiento. Su objetivo es reforzar la competitividad europea o contribuir a la solución de problemas importantes a través de la movilización de una masa crítica de investigaciones y recursos de desarrollo tecnológico y talentos existentes en Europa.

Cada uno de estos proyectos integrados ha de precisar los objetivos en términos de conocimiento científico y tecnológico y ha de resultar aplicable a productos, procesos o servicios.

Los participantes en estos proyectos constituyen un consorcio que se organiza alrededor de dos grupos:

- El llamado core group o núcleo central, a quienes corresponde la ejecución del proyecto. Debe incluir miembros de al menos tres países diferentes, bien de Estados miembros o países asociados al Programa Marco, de los cuales al menos dos han de ser de Estados miembros. En la práctica, el número de participantes no debería ser menor de seis y en ocasiones más de seis, teniendo en cuenta la experiencia y el interés ya mostrado en esta posibilidad.

- El llamado associated partners o miembros asociados, a quienes corresponde una función de apoyo de los anteriores en actividades específicas ${ }^{41}$.

La dimensión del proyecto, esto es, el número de miembros y el presupuesto, variará en función del tema y de los objetivos, conforme a la masa crítica necesaria para obtener los resultados en un tiempo razonable y en las mejores condiciones posibles.

La Comisión selecciona los proyectos sobre la base de una llamada pública para las propuestas ${ }^{42}$ y una posterior evaluación de las mismas ${ }^{43}$

41 Estas actividades pueden haber sido planteadas desde el principio, pero que pueden ser definidas precisamente justo después del lanzamiento del proyecto; o también pueden ser actividades nuevas que se consideren necesarias, de acuerdo con la reorientación del proyecto, con el fin de alcanzar los objetivos del proyecto.

42 Las propuestas deben contener los siguientes elementos: -los objetivos y resultados que se esperan, así como el impacto que pueda causar; -las líneas principales y el tiempo de ejecución, indicando los vínculos entre las distintas partes; -las funciones, actividades y competencia de cada participante; un plan para la gestión y la difusión de información y para la explotación de los resultados; un presupuesto global estimado.

43 La Comisión se basa para la evaluación de las propuestas en el método conocido como peer review, siguiendo, entre otros, los siguientes criterios: -la excelencia científica y tec- 
b) Las llamadas Redes de Excelencia ${ }^{44}$

El objetivo de estas redes consiste en reforzar la excelencia científica y tecnológica de Europa a través de una progresiva y duradera integración de las capacidades de investigación existentes o que puedan presentarse en Europa.

Cada una de las redes pretende avanzar en el conocimiento sobre temas principales ya definidos y que deben cubrir una variedad de asuntos o argumentos, de diversas asignaturas o disciplinas interrelacionados. Y con el fin de crear un «Centro virtual de excelencia», cada red aplica un «programa de actividades combinado».

En cuanto a las características de estas redes de excelencia, lo primero que debe conocerse es ¿en qué consiste la excelencia de la red?:

La excelencia de una red es entendida como la excelencia de sus miembros, de la red en su conjunto y de su programa de actividades. La integración de las capacidades de investigación y las actividades de sus miembros es una característica esencial de las redes de excelencia.

En segundo lugar, ¿̇cuál es su estructura y como se organiza?:

La red se constituye alrededor de un core group de participantes, a los que se pueden unir otros miembros asociados que, en todo caso, contribuyen al establecimiento de actividades específicas del programa de actividades, a menudo en apoyo de uno o varios de los miembros principales. Estos últimos son los que, en todo caso, integran una parte considerable, o incluso la totalidad, de las capacidades de investigación en las áreas pertinentes. El core group de cada red debe comprender miembros de al menos tres países distintos, de Estados miembros o países asociados, de los cuales al menos dos han de pertenecer a algún Estado miembro.

Los miembros principales de una red de excelencia son entidades existentes sin centros de investigación, Universidades, o grandes y pequeñas empresas, que se encuentren en situación de integrar sus capacidades y actividades.

Los miembros asociados son otro tipo de organizaciones tales como aquellas que tengan competencias específicas en gestión de la información y el conocimiento, difusión y transmisión, así como organizaciones interesadas en el uso de los resultados.

El «programa de actividades combinado» se centra en las actividades de investigación que se integran. También incluye las actividades

nológica del proyecto, incluido el carácter innovador de la investigación; - el impacto esperado del proyecto en los términos de los objetivos especificados en la parte pertinente del Programa Marco; -la capacidad del consorcio para concluir el proyecto con éxito, en términos de recursos, composición y habilidades para la gestión; etc.

44 Puede verse el texto en inglés (Provisions for implementing Networks of Excellence) en la página web: http://www.sost.cdti.es/programaVI_1.htm. 
de apoyo a la integración y las actividades que pretenden extender la excelencia y capacidad fuera de la red.

Las actividades de investigación integradas están destinadas a avanzar en el conocimiento en relación con el tema principal de la red, y no a producir resultados predefinidos.

Las actividades de apoyo a la integración generalmente comprenderán: -el desarrollo y uso de los medios electrónicos de información y comunicación avanzados y el desarrollo de los métodos de trabajo virtuales e interactivos; -el cambio de personal a corto, medio y largo plazo y la apertura de posiciones a investigadores procedentes de otras redes; el desarrollo y uso de las infraestructuras combinadas de investigación y la adaptación de los equipos existentes para su uso compartido.

Las actividades encaminadas a extender la excelencia y capacidades de la red incluyen, entre otros aspectos, la formación de los investigadores de organizaciones que no pertenecen a la red; los servicios de apoyo de la innovación tecnológica; etc.

Las Redes de Excelencia son también seleccionadas por la Comisión sobre la base de una llamada pública de propuestas y posterior evaluación de las mismas.

La idea es la creación de un «mapa de la excelencia en Europa». Lo cierto es que el concepto de «excelencia» aún no está definido y su aplicación y futuro no es del todo clara. En todo caso, debe insistirse en que la excelencia está ligada a las personas y no a las Instituciones. Por otra parte, la participación de las empresas, Universidades y Centros de Investigación debería no sólo permitirse, sino asegurarse efectivamente a través de mecanismos concretos ${ }^{45}$.

c) La participación en los programas nacionales realizados conjuntamente, de acuerdo con el artículo 169 TUE que permite a la Comisión adoptar iniciativas para promover una coordinación entre éstos

A ello se refiere la Comunicación de la Comisión al Consejo y al Parlamento Europeo de 30 de mayo de $2001^{46}$.

El artículo 169 del Tratado abre la posibilidad a la Comunidad, en aplicación del Programa Marco, de atender las necesidades de participación en programas de investigación y desarrollo emprendidos por varios Estados miembros.

45 Además, las «redes de excelencia» están restringidas a las áreas temáticas señaladas y sería conveniente su extensión a temas no incluidos en las áreas prioritarias.

46 Comunicación titulada «The Framework Programme and the European Research Area: application of Article 169 and networking of national programes», COM (2001) $282 \mathrm{fi}$ nal. 
Este instrumento es de distinta naturaleza que los dos anteriores. Su aplicación presupone una acción anterior por los Estados miembros, las autoridades regionales o los organismos nacionales de investigación. Por ello sólo puede ser utilizado bajo ciertas condiciones. Las propuestas del Programa Marco y de los programas específicos permiten la posibilidad de usar el artículo 169 en las áreas temáticas prioritarias de investigación. El artículo 169 no ha sido utilizado nunca antes de la aplicación del Programa Marco y no se tiene ninguna experiencia de cómo se podría contribuir al Programa. Sin embargo, la utilización de este artículo combinado con otros instrumentos parece, a priori, adecuado para la creación del EEI; es un medio efectivo para la estimulación de la cooperación entre las regiones de diferentes Estados miembros o entre varios países de Europa.

\section{LOS PROGRAMAS ESPECÍ́fICOS DEL PROGRAMA MARCO 2002-2006}

El documento descriptivo de cómo se va a implementar el PM viene definido por la Comisión en su propuesta de 30 de mayo de 2001 sobre los «Programas específicos del Programa Marco 2002-2006» ${ }^{47}$.

Son en concreto cinco programas específicos: uno para los dos ejes «Integrar la investigación europea» ${ }^{48} \mathrm{y}$ «Reforzar las bases del EEI», otro para el eje «Estructurar el EEI» ${ }^{49}$ y otro para las actividades del «Centro Común de Investigación europeo» ${ }^{50}$. Los otros dos se dedican al Programa Marco EURATOM.

47 Versión en inglés consultada: Proposals for Council Decisions concerning the especific programes implementing the Framework Programme 2002-2006 of the European Community for research, technological development and demostration activities, COM (2001) 279, de 30 de mayo de 2001.

Y la propuesta modificada para la Decisión del Consejo (versión en inglés), COM (2002) 43 final, de 30 de enero de 2002.

48 Este programa define las prioridades científicas comunes sobre las que la Comisión concentrará el apoyo financiero y prevé una importante iniciativa nueva diseñada para ayudar a la creación de redes de programas nacionales de investigación en los Estados miembros.

49 A través de este programa se pretende paliar las deficiencias estructurales de la investigación europea mediante una mejor integración de la investigación y la innovación, una mayor movilidad de los investigadores, la planificación coordinada y el acceso a las infraestructuras de investigación y otras acciones como el fomento de la ética en la ciencia, la lucha contra la «fuga de cerebros» y la sensibilización de la población hacia la ciencia.

50 Dos programas reservados a este Centro de Investigación: el primero, referido a la seguridad alimentaria y la salud, el medio ambiente y el desarrollo sostenible, la previsión tecnológica, la metrología, combatir el fraude, el seguimiento/predicción de los desastres naturales y la seguridad de los datos; el segundo, referido a las actividades del Centro Común de Investigación en el ámbito nuclear. 


\section{DOS AÑOS DESPUÉS DEL CONSEJO EUROPEO DE LISBOA. NECESIDAD DE REFORZAR EL EEI}

La creación del EEI es el elemento clave de la estrategia definida en Lisboa en marzo de 2000, con el fin de crear un verdadero «mercado interior de la investigación», a través de la coordinación de las políticas nacionales de investigación.

A pesar de la toma de conciencia de los Estados miembros de la dimensión europea de la investigación, dos años después del Consejo de Lisboa los resultados obtenidos, con ser buenos porque reflejan las iniciativas nacionales y los esfuerzos invertidos, no son, ni mucho menos, los esperados. Los obstáculos permanecen; el proyecto del EEI progresa, pero de modo desigual según los ámbitos y las dimensiones. Es por ello por lo que la Comisión Europea analiza el desarrollo del proyecto y trata de impulsarlo destacando obstáculos y objetivos en su Comunicación «El Espacio Europeo de Investigación: Un nuevo impulso. Reforzar, reorientar, abrir nuevas perspectivas» ${ }^{51}$.

En esta Comunicación, la Comisión advierte de los principales problemas con los que se enfrenta el proyecto que son el nivel insuficiente de los esfuerzos financieros en este ámbito; la falta de un entorno que estimule la investigación y la explotación de sus resultados; y la fragmentación de las actividades y la dispersión de los recursos.

Y como objetivos generales señala los siguientes:

1.- Reforzar la participación de los Estados miembros y la movilización de las actividades nacionales.

2.- Aumentar el impacto de las acciones emprendidas.

3.- Consolidar el marco conceptual y político dentro del cual se ejecuta el proyecto.

Uno de los aspectos fundamentales que considera es el referido al porcentaje del PIB que dedica la UE a la investigación y al desarrollo, tan sólo el 1,9\%, frente al 2,7\% alcanzado por los Estados Unidos y el $3 \%$ de Japón. En este sentido, la Comisión plantea el objetivo de alcanzar el $3 \%$ del PIB de la Unión para 2010 en la Comunicación «Más investigación para Europa - Objetivo: $3 \%$ del PIB» ${ }^{52}$. En ella se advierte de la necesidad de políticas diferenciadas, pero coordinadas, dada la diversidad de las situaciones regionales y nacionales en Europa y que generen el impulso necesario para alcanzar el objetivo del $3 \%$. Y confirma la importancia no sólo de aumentar la eficacia del sistema eu-

51 COM (2002) 565 final, 16.10.2002.

52 COM (2002) 499 de 11.9.2002. Objetivo ya planteado en el Consejo Europeo de Barcelona de 2002. 
ropeo de $\mathrm{I}+\mathrm{D}$ e innovación, sino también de solucionar la escasez de inversiones en I $+\mathrm{D}$ en la UE. Para ello los esfuerzos han de ser conjuntos y deben participar las instituciones europeas, todos los Estados miembros y los países candidatos, así como el sector empresarial.

Del examen que realiza la Comisión extrae como conclusión que efectivamente el proyecto del EEI ha modificado el entorno de la investigación y de las políticas de investigación en Europa, pero que existen obstáculos de carácter estructural que reducen el alcance de los resultados obtenidos por lo que los esfuerzos deben intensificarse ${ }^{53}$. De modo que como nuevas perspectivas a considerar debe, en primer lugar, corregirse el fallo de la coordinación de las políticas nacionales de investigación, al ser esta coordinación la base para el desarrollo del EEI; en segundo lugar, aprovechar mejor los instrumentos jurídicos de que se dispone (directivas, reglamentos, recomendaciones); en tercer lugar, aprovechar las iniciativas de los grandes agentes de cooperación europea; y, en cuarto lugar, admitir la plena participación en el proyecto de EEI de los países candidatos ${ }^{54}$.

\section{REPERCUSIONES DE LA POLÍTICA COMÚN DE IDT PARA EL DERECHO ADMINISTRATIVO}

Toda política común tiene sus repercusiones para las políticas internas de cada Estado miembro. La política de IDT afecta, desde luego, a la normativa interna y al Derecho administrativo en varios aspectos.

De ellos debe destacarse:

\section{LA MODERNIZACIÓN DE LAS ADMINISTRACIONES PÚBLICAS}

En primer lugar, la aplicación real de toda la normativa de incorporación y uso de las nuevas tecnologías por el sector público. Éste es quizá un problema de adaptación del funcionamiento normal de las Administraciones Públicas, pero, sobre todo, es un problema financie-

53 Somete a análisis: la evaluación comparativa de las políticas de investigación; la cartografía de la excelencia; la movilidad de los investigadores; las infraestructuras de investigación; la conexión en red de los programas nacionales de investigación; el refuerzo de la inversión privada en investigación; la propiedad intelectual; la red electrónica transeuropea de investigación; la dimensión internacional del EEI; la dimensión regional del EEI; y la Ciencia y sociedad.

54 Si bien, teniendo en cuenta la situación de los sistemas de investigación de estos países y la falta de medios, su integración en el EEI es más teórica que práctica, lo que exige ayudas concretas por parte de la UE y de los Estados miembros para hacer efectiva la participación. 
ro, pues la «modernización» de las Administraciones Públicas exige una inversión importante en medios materiales y en procesos de aprendizaje y manejo del personal al servicio de las Administraciones Públicas. Ésta es la base que permite el desarrollo de la política en IDT.

Como expresamente ha destacado la Comisión Europea, la modernización del sector público ya no consiste en introducir nuevas tecnologías, sino que, además, es necesario «modificar las normas y las prácticas de trabajo para cosechar los beneficios que aporta la tecnología ${ }^{55}$. Por ello insiste en que las Instituciones comunitarias y las Administraciones Públicas nacionales han de utilizar las tecnologías de la información para desarrollar servicios eficientes al servicio de las empresas y los ciudadanos europeos.

En concreto, las Administraciones Públicas deben:

1.- Desarrollar servicios basados en Internet para mejorar el acceso de los ciudadanos y las empresas a la información y los servicios públicos.

2.- Utilizar Internet para mejorar la transparencia de la Administración Pública e implicar a los ciudadanos y las empresas en la toma de decisiones de manera interactiva.

3.- Hacer posible la plena explotación de las tecnologías digitales dentro de las Administraciones, incluyendo la utilización de programas de fuente abierta y firmas electrónicas.

4.-Crear mercados electrónicos para la contratación electrónica, partiendo del nuevo marco comunitario sobre contratación pública.

\section{LA ORGANIZACIÓN ADMINISTRATIVA ${ }^{56}$}

La implementación del EEI afecta a las autoridades de ciencia y tecnología y no sólo a la Comisión Europea. La política comunitaria de IDT exige que en los Estados miembros existan unos organismos especializados en la materia, a través de los cuales se ejecute esta política con coherencia y en coordinación con las políticas nacionales. De ello se ocupa el Ministerio de Ciencia y Tecnología y los Organismos públicos de investigación.

55 En la Comunicación «e-Europe 2002»(COM/2001/0140 final/). En esta Comunicación destaca la Comisión que las Administraciones Públicas son más lentas en introducir los servicios en línea; así, la contratación electrónica aún no es una realidad aparte de la simple aceptación de ofertas por correo electrónico y no es fácil obtener en todos los Estados miembros información sobre el sector público crucial para los servicios de valor añadido.

56 Véase en este mismo número el artículo sobre «Organización administrativa de la ciencia y la tecnología: el Ministerio de Ciencia y Tecnología y los organismos públicos de investigación», de Estanislao ARANA GARCía. 
Además, las Comunidades Autónomas también disponen de una política de fomento que incluye medidas destinadas al desarrollo de la investigación y el proceso tecnológico.

Ello exige un doble esfuerzo de coordinación: de un lado, el esfuerzo de coordinación propio de la política común de IDT, con las políticas nacionales; pero, de otro lado, es fundamental la coordinación de la política estatal con las políticas autonómicas.

Debe insistirse en la necesidad de una ejecución coordinada de los programas de investigación nacionales y europeos. En este sentido, las Administraciones nacionales de Investigación de los Estados miembros recomiendan la adopción del principio «de apertura mutua de los programas nacionales» y adoptar las medidas necesarias para garantizar su aplicación práctica. Para ello, se exigen unos mecanismos de información recíproca y un dispositivo de información general sobre los objetivos y contenidos de los programas y las condiciones de participación y admisión en los mismos.

\section{LA EDUCACIÓN SUPERIOR: LA FUNCIÓN DE LA UNIVERSIDAD}

La realización del EEI es una responsabilidad compartida entre la Comunidad y los Estados miembros; de ahí la necesidad de un marco legal referido a:

- La libre circulación de investigadores: lo que afecta a la normativa interna, en cuanto a la movilidad del investigador nacional.

- La protección intelectual.

- La transferencia de resultados.

Con respeto al principio de subsidiariedad, en su más amplio sentido, esto es, las acciones deben iniciarse en el ámbito en el que pueden realizarse con más eficacia; un EEI eficaz implica definir con nitidez cuáles son las funciones del sector público y privado, así como lo que debe hacerse en los ámbitos regional, nacional, europeo e internacional.

Como parte integrante del EEI, algunas acciones del VI PM merecen en este apartado reseñarse, pues constituyen un ámbito importante que afecta a nuestro Derecho administrativo.

Así, deseo referirme al ámbito de la educación, teniendo en cuenta el principio de la tradición europea en esta materia: la educación es un servicio público y la calidad se erige en pilar esencial.

Y expresamente la Educación Superior, en tanto en cuanto uno de los objetivos básicos para la realización del EEI es el intercambio Uni- 
versidad-Empresa, lo que influye necesariamente en la forma de concebir la Universidad y su modo de actuar y, por lo tanto, en la normativa que la configura.

En este sentido, desde la Unión Europea, se exige el desarrollo de una Universidad dotada de autonomía con responsabilidad, una autonomía que sea suficiente, una normativa ligera y propicia y una financiación adecuada, de manera que sea útil para competir y cooperar y mejorar la calidad de la enseñanza superior ${ }^{57}$.

La necesidad de un esfuerzo público de investigación, así como también la promoción de la inversión privada, a través de apoyos indirectos, fundamentalmente de medidas fiscales.

El papel de las Universidades en este proceso es fundamental, pues de ellas se deriva el conocimiento científico y tecnológico, la difusión del mismo, la reflexión crítica sobre la evolución y los efectos que éste tiene sobre el sistema de ciencia y tecnología y, en definitiva, sobre el ciudadano europeo; además de su contribución como formadora de los futuros investigadores europeos.

Como expresamente ha señalado la Conferencia de Rectores de las Universidades Españolas (CRUE), el «rol multifacético» que corresponde a la Universidad es lo que determina su especial sensibilidad frente a todas las actuaciones de política científica y tecnológica promovidas por los poderes públicos y, en particular, por la Unión Europea cuyo impacto sobre el futuro de las Universidades europeas será progresivamente mayor ${ }^{58}$.

57 Lo que significa que las Universidades puedan diseñar sus estrategias, elegir sus prioridades en cuanto a docencia e investigación, asignar sus recursos, desarrollar sus currícula y fijar sus criterios de admisión de estudiantes y profesores.

En este sentido, la Convención de Instituciones Europeas de Enseñanza Superior, reunida en Salamanca los días 29 y 30 de Marzo de 2001, se pronuncia en apoyo a la creación de un Espacio Europeo de la Enseñanza Superior y consideran que la creación en Salamanca de la Asociación Europea de la Universidad, por su valor simbólico y también práctico, servirá para transmitir con más fuerza su mensaje a los Gobiernos y las sociedades.

Además, dado que la investigación es el motor de la enseñanza superior, la creación de un Espacio Europeo de la Enseñanza Superior y la creación del Espacio Europeo de la Investigación deben completarse.

En cuanto al significado de la calidad como pilar esencial, implica que el Espacio Europeo de la Enseñanza Superior se desarrolle sobre unos valores académicos fundamentales. La calidad exige un equilibrio entre innovación y tradición, excelencia académica y pertinencia social y económica, coherencia de los currícula y libre elección del estudiante; comprende la función docente e investigadora pero también la dirección y la administración, la capacidad de respuesta a las necesidades de los estudiantes así como la prestación de otros servicios además de los educativos. La calidad debe demostrarse y garantizarse para que sea apreciada por los estudiantes, los responsables y toda la sociedad del país, de Europa y del resto del mundo.

58 Véase en el documento sobre la «Posición común de las Universidades Españolas frente a la propuesta de la Comisión relativa al VI PM», op. cit. 
Son varias las barreras que frenan el feliz camino de la política de IDT hacia la consagración de un EEI, en este ámbito concreto ${ }^{59}$ de la educación universitaria:

1.- Una de ellas es la dificultad de la movilidad de los investigadores en Europa, provocada, entre otros obstáculos, por la falta de familiaridad de los investigadores europeos con las «culturas» de investigadores de otros países y la falta de adaptación hacia las mismas.

Ello exigiría procesos de formación en el centro de origen sobre las «culturas» de los países donde radica el centro de destino, con el fin de aumentar la movilidad de los investigadores en Europa, sobre todo tratándose de países de nueva incorporación a la Unión Europea.

Además, la diversidad de idiomas, de sistemas nacionales, de tipos y perfiles de institución, existentes en Europa, no debe traducirse en un freno al desarrollo de un Espacio Europeo de la Enseñanza Superior, complementario del EEI, antes al contrario, la solución está en considerar la diversidad como una cualidad positiva y no como un motivo de exclusión; es decir, tal diversidad debe integrarse con eficiencia teniendo en cuenta los principios comunes a los países dentro de una determinada disciplina y garantizar la cohesión mínima necesaria para no paralizar el progreso hacia la compatibilidad.

Unido a lo anterior, es necesario establecer un sistema que facilite la incorporación de investigadores de otros países dentro del EEI. En nuestro sistema se exige la revalidación del título superior y del título de doctorado, lo que conlleva trámites administrativos de una duración de al menos un año o año y medio, lo que dificulta o impide cualquier incorporación.

Por ello la simplificación del sistema nacional de homologación de Títulos Superiores en Europa, a través de una validez inmediata, permitiría la movilidad de investigadores y de funcionarios de la Administración Pública.

Como instrumentos que pueden servir para favorecer la movilidad de los investigadores europeos en la Comunidad, destaca la Comisión, los incentivos financieros y los instrumentos jurídicos ${ }^{60}$.

59 No olvidemos que la política común de IDT tiene como destinatarios los Centros nacionales de investigación, las empresas y las Universidades.

${ }^{60}$ Véase la Comunicación «El Espacio Europeo de Investigación: Un nuevo impulso. Reforzar, reorientar, abrir nuevas perspectivas», op. cit.

Se han ampliado las becas a toda la carrera de los investigadores y también una prolongación de su duración. Por otro lado, se han puesto en marcha otras iniciativas en otros planos dentro de un proceso de coordinación de las políticas de los Estados miembros sobre este tema, así: la creación de una red europea de centros de movilidad dirigidos a proporcionar a los investigadores ayuda práctica y asistencia; la creación de un sistema de información electrónico sobre las oportunidades de empleo para los investigadores, y las disposiciones jurídicas y administrativas en los países de la Unión denominado «Researcher's Mobility Web Portal», operativo a principios de 2003. 
2.- Pero también aparecen obstáculos de carácter administrativo derivados de la aplicación nacional de las Directivas comunitarias sobre la libre circulación y derecho de establecimiento, la protección social o pensión, que no siempre es fácil.

3.- La falta de una dimensión europea de las carreras científicas.

Es necesario cuanto antes asumir que la competencia es útil para mejorar la calidad de la Enseñanza Superior. Sin embargo, las Universidades de ciertos países europeos aún no están en condiciones de poder competir en condiciones de igualdad y ello implica una «fuga de cerebros» hacia otros países europeos. Con las «Redes de Excelencia» se pretende evitar este fenómeno.

El Comité Económico y Social, en su Dictamen sobre la Comunicación de la Comisión acerca de la creación de un $\mathrm{EEI}^{61}$, ha planteado la posibilidad de creación de una Universidad europea, financiada y gestionada en común por los «centros de excelencia» europeos, con el fin de realizar el ideal de la investigación y la enseñanza a nivel europeo, gracias a la colaboración entre los mejores institutos de investigación europeos, que competirían al mismo tiempo entre ellos.

Una Universidad europea debe contribuir a la introducción de una dimensión europea en las carreras científicas y a convertir el territorio europeo en atractivo para los investigadores del resto del mundo.

A pesar de las iniciativas comunitarias y el significado otorgado a las Universidades en este contexto, es necesario que la Unión Europea continúe con un creciente apoyo a las mismas, y se refleje en el aumento de la movilidad, en el reconocimiento de títulos y diplomas; y que los Estados miembros y las Universidades adopten las medidas pertinentes para superar los obstáculos jurídicos y administrativos que dificultan la movilidad.

61 CES 595/2000, 24 de mayo de 2000. 